Revue d'histoire de l'Amérique française

BHS REVUE D.HISTOIRE DE L'AMÉRIQUE FRANÇAISE

\title{
Histoire documentaire du Canada au milieu du XVIIIe siècle
}

\section{Roland Lamontagne}

Volume 18, numéro 2, septembre 1964

URI : https://id.erudit.org/iderudit/302359ar

DOI : https://doi.org/10.7202/302359ar

Aller au sommaire du numéro

Éditeur(s)

Institut d'histoire de l'Amérique française

ISSN

0035-2357 (imprimé)

1492-1383 (numérique)

Découvrir la revue

Citer cet article

Lamontagne, R. (1964). Histoire documentaire du Canada au milieu du XVIIIe siècle. Revue d'histoire de l'Amérique française, 18(2), 176-195.

https://doi.org/10.7202/302359ar d'utilisation que vous pouvez consulter en ligne.

https://apropos.erudit.org/fr/usagers/politique-dutilisation/ 


\section{HISTOIRE DOCUMENTAIRE DU CANADA AU MILIEU DU XVIIIe SIËCLE}

L'histoire est une discipline qui se fonde sur les sources manuscrites et imprimées. Elle a pour but d'évoquer le passé de façon aussi objective que possible. Les ressources de la recherche par groupe de travail, de la codification numérique et de la mécanographie devraient être mises à profit pour recueillir le plus grand nombre de témoignages et de données se rapportant à un sujet d'étude. L'analyse approfondie de la mise en œuvre des facteurs de colonisation en Amérique nécessiterait l'apport d'un carrefour interdisciplinaire.

Le choix et l'utilisation des documents recèlent déjà une interprétation. Dans le cadre d'un sujet de recherche même restreint, les résultats demeurent largement approximatifs. Le nombre de variables dans les faits du passé reste indéterminé. A partir de conclusions susceptibles d'être contestées, il semble qu'une extrapolation du passé pour expliquer le présent risque fort d'être abusive. Compte tenu de ces limites de la connaissance historique, les idées générales d'ordre explicatif devraient être exprimées avec toutes les réserves et la prudence méthodologique qu'exige le souci de l'objectivité.

Ce fragment d'histoire fait revivre des écrits relatifs au plan d'action de Barrin de La Galissonière. Il comporte également l'examen d'une double série de faits touchant aux négociations franco-britanniques et à la marine, agent de civilisation technique. Le terme de technique est ici employé dans un sens restreint d'activités portant sur les matériaux et les ressources naturelles et visant une utilité pratique. ${ }^{1}$

1 François Russo, "Histoire des techniques", Le Monde (Sélection hebdomadaire du 20 au 26 février 1964), No 801, 7. 


\section{LE PLAN DE DÉVELOPPEMENT DE BARRIN DE LA GALISSONIERE}

La présence de La Galissonière en Nouvelle-France est due à un acte de guerre. Le 6 juin 1747, le comte de Maurepas, ministre de la Marine, informe Barrin de La Galissonière de la "malheureuse aventure" qui vient d'arriver au marquis de La Jonquière qui s'en allait rapatrier la flotte du duc d'Anville.

Ce document émané du ministère de la Marine illustre le style des dépêches de Maurepas et présente le récit des événements, à l'époque de la nomination du gouverneur intérimaire Barrin de La Galissonière.

A M. le Comte de La Galissonière. ${ }^{2}$

A Paris le 6 juin 1747.

Il se presente pour vous une occasion fort interessante de donner de nouvelles preuves de votre zele et de votre attachement pour le service du Roy, et je n'ai point hezité à assûrer S. M. que vous en profiterés avec tout l'empressement qu'elle doit attendre d'un officier tel que vous. Voici de quoy il est question.

Vous aurés sans doute appris les facheuses nouvelles qui sont venues au sujet de l'Escadre de M. de la Jonquière. Les Anglois embarqués sur le navire le grand Scypion de la Rochelle repris par un corsaire de St Malo et conduit a Morlaix, annoncent la prise du Serieux, de la Gloire et de l'Emeraude. Un autre navire de notre flotte relaché a la Corogne ne parle que d'une fregate de 40 canons. Mais tout s'accorde sur la grande Superiorité de l'Escadre Angloise; de maniere qu'il faut s'attendre que quelque chose qu'ait pu faire la nôtre, elle aura été accablée par le nombre.

Dans la necessité de prevenir autant qu'il sera possible, les suittes que cette malheureuse aventure pourroit avoir par raport au Canada, le Roy s'est determiné à envoyer de nouveaux secours à cette Colonie; et comme la diligence est ce qu'il y a de

2 AC, B, 86: 335-339. 
plus essentiel, S. M. destine ses $V^{\mathbf{x}}$ le Northumberland ${ }^{2 b}$ et l'Alcyon avec la flute la Gironde à porter ces secours.

Je prescris en conséquence à $M$. de Givry de tirer des Magazins du port les armes et les munitions de guerre qui s'y trouveront pour remplacer ce qui en avait été chargé dans la flotte de M. de la Jonquiere, de prendre chés les marchands de Rochefort ce qui pourra s'y trouver de marchandises propres pour le Canada, des vins et eaux de vie dans les Magazins du Munitionnaire, et une nouvelle provision de sel des fournisseurs ordinaires.

Je fais prendre aussi tout le chargement du navire le Grand Scypion conduit à Morlaix pour être envoyé directement par un bon corsaire de St Malo à Quebec.

J'ai pris des mesures pour faire acheter sans retardement à Bordeaux et a la Rochelle des marchandises propres pour le Canada.

Je compte qu'on pourra aussi avoir 12 à 1500 fusils des magazins de la terre.

Et j'envoye des ordres dans tous les ports de la Côte d'Espagne pour en faire repartir les Navires de la flotte qui pourront y avoir relaché.

Mais le secours le plus important et dont la Colonie se trouve avoir le plus de besoin c'est un chef en état de la conduire, et de la deffendre. Il y a tant à craindre qu'il ne soit arrivé à $M$. de la Jonquière quelque accident qui l'empeche de s'y rendre. Dans l'incertitude ou l'on est à cet egard le Roy a jugé à propos de vous choisir pour y suppléer. Sa Majesté veut qu'à cet effet, vous vous embarquiés sur le Northumberland, et elle m'a ordonné de vous expedier des lettres patentes qui

${ }^{2 b}$ Les volumes de la série Marine $\mathrm{B}^{4}$ (Campagnes), de 1744 à 1779, correspondent à la carrière du Northumberland sous pavillon français. Dans son ouvrage THE ROYAL NAVY, vol. 3, p. 274, Wm. Laird Clowes relate les circonstances de la capture du Northumberland, en 1744.

D'après le Répertoire des navires de guerre français de Pierre Le Conte, le Northumberland fut transformé en flûte en 1779 et renommé l'Atlas. Un autre vaisseau français porta le nom de Northumberland de 1779 à 1794 . 
vous donnent au defaut de $M$. de la Jonquiere le commandement g'nal de la Nouvelle-France, avec l'autorité, les pouvoirs, les honneurs et les appointemens de Gouverneur

Si M. de la Jonquiere se rend en Canada, vous pourrés vous en revenir avec les Vaisseaux. Supposé qu'il lui soit arrivé accident vous prendrés le commandement general de la Colonie, je vous enverray, si vous voulés, des provisions en forme de gouverneur lt g'nal, et vous serés le maître de n'y rester que 2 ans, S.M. vous laissant dès à present toute liberté sur cela. Mais dans l'un et l'autre cas Elle aura en partre consideration la soumission avec laquelle vous devés vous livrer à cette destination; Et la façon de penser que vous me connoissés sur votre compte doit vous tranquilliser sur l'attention que j'aurai à faire valoir les services que vous pourrés y rendre.

Pour qu'il n'y ait pas de temps perdu dans les arrangements a prendre pour accellerer votre depart et que je ne sois pas obligé d'envoyer de nouveaux ordres sur toutes les difficultés qui pourroient survenir je prends deux precautions: l'une de faire partir dès demain pour Rochefort M. Delalanne qui vous expliquera mes intentions sur les divers objets, et qui par les connoissances partres qu'il a de ce qui regarde la Colonie de Canada pourra contribuer à tous ces arrangemens; Et l'autre de vous mettre à portée de les faire faire comme vous les jugerés plus convenables, au moyen de l'ordre precis qui est envoyé à M. de Barrailh et a M. de Givry, de donner tous ceux que vous demanderés. Vous trouverés cy joint copie de l'ordre partre adressé à M. de Barrailh.

Au surplus si l'Alcyon n'etoit pas en etat de faire la Campagne de Canada, il faudra le remplacer par l'Aurore, en faisant passer $M^{\text {rs }}$ Gaulette et Desherbiers respectivement de l'un à l'autre. Je suis, M., très parfaitement à vous,

Maurepas.

La Galissonière avait sollicité des fonds métropolitains. Il est vrai que ses prises de position s'inscrivent dans le cadre 
du mercantilisme: la colonie doit contribuer à l'expansion du commerce de la France. En 1747, lorsque Barrin de La Galissonière aborde au Canada, le traité d'Aix-la-Chapelle n'est pas encore signé. L'année suivante, la Nouvelle-France aura la satisfaction de reprendre Louisbourg. Les frontières de l'Acadie et de l'Ohio ne seront pas nettement fixées. La commission internationale, composée de La Galissonière et de Silhouette ainsi que de Mildmay et de Shirley, ne réglera pas le litige. Son objectif consistait à assurer un échange de vues et d'informations qui permettait à chacune des métropoles d'apprécier la politique frontalière et d'en préparer une nouvelle qui tienne compte des intérêts réciproques.

La Galissonière se révèle un administrateur du calibre de l'intendant Talon, en raison de son plan de mise en valeur de la colonie. Il disposait de la liberté d'esprit nécessaire à la formation d'un programme qu'il expose dans cette lettre à Maurepas et qu'il précisera dans son mémoire de décembre 1750 .

A Québec le 24 août $1747 .^{3}$

Monseigneur,

La communication que Monsieur Hocquart m'a donnée de vos dépêches sur les finances étoit en quelque façon indispensable, puisqu'une grande partie des dépenses extraordinaires se font sur mes ordres, et à cette occasion je crois être obligé de vous dire quelque chose en général sur cette matière.

Les dépenses sont sans doute très considérables et surpassent vraisemblablement le produit, mais je vous prie de vouloir bien observer

$1^{\circ}$ qu'il y a en France beaucoup de places frontières qui ne produisent rien du tout, qui ont cependant été bâties à grands frais et qui sont entretenues et gardées avec grande dépense.

$2^{\circ}$ qu'on ne peut négliger ce pays ci sans perdre pour jamais avec lui tous nos établissements de pêche, branche de commerce très lucrative.

${ }^{3} \mathrm{AC}$, CIIA, 87-2: 204-210. 
$3^{\circ}$ que les avantages que nous perdrions devant passer à nos perpétuels ennemis. Il y aurait lieu de commenter ce qualificatif à la lumière de PACEM IN TERRIS. La perte que nous ferions doit être censée double ou triple.

$4^{\circ}$ que la navigation de ce pays ci forme beaucoup les matelots et en détruit très peu, celle des Isles de l'Amérique fait tout le contraire.

$5^{\circ}$ que les principales denrées de ce pays ci, comme le bled, le poisson, les chanvres étant d'un usage nécessaire à la vie et toute la consommation n'a point de bornes, son commerce sera de plus en plus solide et ira toujours en augmentant, celui des Isles au contraire doit diminuer au bout d'un tems.

$6^{\circ}$ que ce pays contient desjà un assez grand peuple, propre à la fatigue, à la guerre, et à la navigation, peuple qu'on perdrait pour la plus grande partie avec le pays, et qui avec le temps fortifieroit nos ennemis, comme il est à craindre qu'il n'arrive dans peu à l'Acadie.

$7^{\circ}$ que si les autres colonies produisent plus de richesses, celle-ci produit des hommes, richesse bien plus estimable pour un grand Roy que le sucre et l'indigo ou si l'on veut tout l'or des Indes.

$8^{\circ}$ que la fécondité est telle qu'elle peut remplacer en partie la perte immense de monde que nous coutent tous les ans La Martinique et Saint Domingue.

On m'a fait remarquer depuis longtemps que les Canadiens étoient les moins sujets de tous aux maladies qui désolent quelquefois cette dernière isle et j'ai lieu de croire que la meilleure et peut-être la seule façon de la remplir suffisamment de blancs est d'y procurer des établissements à des Canadiens.

Dans cette vue, on pourrait faire passer en Canada beaucoup d'engagés et peu aux Isles et en tirer un peu du Canada pour la Martinique et Saint Domingue.

$9^{\circ}$ qu'outre les François, on peut se flatter d'acquérir un jour la plus grande partie des sauvages à la religion et à l'Etat, quoique cela ne soit encore guères avancé, l'augmentation des peuples 
dans les colonies et surtout celle des troupes y contribueroit beaucoup.

$10^{\circ}$ que la situation de l'Angleterre dans l'Europe et ses forces maritimes ne permettent pas d'y porter la guerre avec espérance de succès qu'ici au contraire tous les avantages de la nature sont pour nous et qu'il ne faut qu'un peu de dépense, pour y détruire plusieurs établissements qui luy sont précieux et qui nuisent beaucoup aux nôtres.

Je pourrois alléguer bien d'autres raisons de conserver et d'aider puissamment cette colonie, je voudrois qu'il me fût aussi aisé de proposer les moyens de le faire sans dépense ou de diminuer celles qui s'y font.

Pour ce dernier article je n'en sais qu'un seul moyen qui est de donner aux cartes et au papier tout le crédit qu'ils peuvent avoir, ce qui ne se peut faire qu'en tirant sans aucune remise, ni difficulté, ni formalité, des lettres de change en un seul et court terme pour tout ce qui s'apportera au trésor; si cela étoit annoncé de bonne heure et que le trésorier tirait pour tout venant sans attendre le mois d'octobre, je suis sûr qu'on s'appercevrait dès la même automne d'une diminution considérable dans le prix des marchandises de France et des denrées $\mathrm{du}$ pays, et par conséquent dans les dépenses $d u$ Roy, diminution qui augmenteroit encore quand on sauroit que les lettres de change auroient été régulièrement acquittées malgré cette facilité.

Je suis persuadé encore que cette avance diminueroit l'empressement pour avoir des lettres de change ce qui prolongeroit d'avantage le crédit du Roy et d'une façon bien moins onéreuse à Sa Majesté et aux particuliers puisque cela seroit purement volontaire.

$$
\text { (...) }
$$

$\mathrm{Au}$ surplus je n'ignore pas la difficulté des fonds en France et surtout pour la Marine dans les circonstances présentes, mais cela n'empêche pas que je ne persiste dans mon opinion.

Je suis avec un très profond respect, Monseigneur, votre très humble et très obéissant serviteur,

La Galissonière 
La Cour ne s'est pas mise à l'écoute de Barrin de La Galissonière, homme simple et droit, qui envisageait l'avenir de l'Amérique française avec une calme décision. Ce gouverneur intérimaire a fait tout ce qui était en son pouvoir pour contribuer à la croissance régulière de la Nouvelle-France. Son mémoire, en date de décembre 1750, traduit l'espoir de stimuler l'immigration au Canada, d'aménager l'industrie et le commerce et d'augmenter les effectifs de troupes. Cette politique apparaît comme une volonté de puissance obstinément tendue vers la protection coloniale, notamment dans les zones critiques de l'Acadie et de l'Ohio.

En dehors des rivalités habituelles entre la France et la Grande-Bretagne, ce que cette politique a de bien fondé souffre du manque d'intérêt de la classe politique française à l'égard de la collectivité canadienne, au XVIII ${ }^{\circ}$ siècle. Pour que puisse se traduire en acte la volonté de jouer dans les affaires d'Europe le rôle de grande puissance, il faut, selon La Galissonière, animer jusqu'aux limites les plus lointaines l'immense corps de l'Amérique française.

Les étapes du mémoire de décembre 1750 sont les suivantes:

1) Définition de l'objectif. - Barrin de La Galissonière insiste sur la nécessité de veiller avec soin à la conservation, à la défense et à l'accroissement de l'Amérique française.

2) Analyse des données. - Dans le but d'éclairer la décision au niveau de la Cour, l'auteur énumère les faiblesses et les avantages de la colonie.

3) Formation d'un modèle logique. — La Galissonière présente un modèle schématisant les principaux éléments de la réalité politique: “Tout ce que l'on vient d'exposer démontre suffisamment, écrit-il, qu'il est de la dernière importance et d'une nécessité absolue de n'omettre aucun moyen et de n'épargner aucune dépense pour assurer la conservation du Canada, puisque ce n'est que par là qu'on peut parvenir à soustraire l'Amérique de l'ambition des Anglois, et que le progrès de leur empire dans cette partie du monde est ce 
qu'il y a de plus capable de leur donner la supériorité en Europe."

4) Dans les hypothèses relatives à chacun des articles, l'auteur examine les conséquences plausibles. Il écrira au sujet de la région des Illinois: "On objectera sans doute que ces profits sont bien éloignés et que peut être s'y trouvera t'il des obstacles imprévus; ... il reste toujours une question, non si l'on doit abandonner ce poste, mais si l'on doit le livrer à l'Angleterre qui en feroit un entrepôt solide pour entreprendre la conquête du Mexique."

5) Au niveau du contrôle des résultats, l'initiative appartient à la Cour. Le fait de ramener à l'unité d'une pensée cohérente la multiplicité du réel est susceptible d'aider le responsable de l'administration à faire converger la faculté du jugement sur ce qui fait l'objet d'une réflexion systématique.

La comparaison des étapes sous-jacentes de ce mémoire à un schéma méthodologique risque peut-être de dépasser la pensée de La Galissonière. Toutefois, dans le cadre d'une tradition de rigueur intellectuelle en voie de formation, à l'exemple des travaux du XVIII ${ }^{\mathrm{e}}$ siècle illustrant une application de la logique à partir des faits, ne découvre-t-on pas dans cet effort de Barrin de La Galissonière une structure de recherche opérationnelle avant la lettre ? Cette discipline consiste fondamentalement à préparer une décision grâce à l'analyse des faits jointe à l'emploi de moyens logiques. $\mathrm{Au} \mathrm{XX}^{\mathrm{e}}$ siècle, à l'aide de l'instrument mathématique, elle s'étend à une grande variété de problèmes de décision ou d'aléas de gestion et de planification. Confrontée aux problèmes complexes de la situation coloniale que décrivait Barrin de La Galissonière, une administration efficace eût pu prendre une décision en connaissance de cause. Cette remarque appelle la précision suivante. A la lumière des concepts élaborés dans un contexte d'interdépendance de l'économie, de la sociologie, de la science politique ou administrative et de la psychologie, la recherche opérationnelle maintenant permet d'explorer les problèmes d'organisation de la vie collective et de déceler les facteurs de perturbation, d'inertie et les lacunes des mécanismes 
de décision; elle contribue à développer le système limité de signes qui traduisent la réalité telle qu'elle est perçue par les témoins du passé. Pour saisir la portée de l'activité de recherche opérationnelle, il convient de situer cette discipline par rapport à la source inépuisable de signes qu'est la réalité elle-même. Ainsi l'observation de l'homme contemporain aide à comprendre les mobiles des actions dont la trace est conservée dans les documents.

Le commun dénominateur des faits historiques, c'est bien la condition humaine. Une puissance de désordre menace nos fragiles structures. L'homme, imprégné d'esprit technique, risque de devenir étranger à soi-même et d'oublier son intériorité. Il aspire à une connaissance unifiée. L'histoire, "maîtresse de vie", peut contribuer à la formation d'une synthèse qui puisse mettre en lumière la signification de l'être humain dans la création.

\section{LES NÉGOCIATIONS FRANCO-BRITANNIQUES}

En guise d'information préliminaire à un aperçu sur les négociations entre la France et l'Angleterre, voici la dépêche que La Galissonière adressait au ministre Rouillé, le 25 juillet 1749 .

A Québec, le 25 juillet $1749 .{ }^{4}$

Monseigneur,

Vous aurez appris par ma dépeche du 26 juin et par les pièces qui y étoient jointes les mouvements que les Anglois, avant même qu'ils sussent la conclusion de la paix avaient commencé à se donner non seulement envers les Acadiens mais envers des habitants françois placés sur des terrains qui sont indubitablement du Canada. Vous avez vu aussi l'envoy que j'ai fait à la rivière de Saint Jean du $\mathrm{S}^{\mathrm{r}}$ de Boishebert et d'un détachement et la lettre que j'ai écrite à M. Mascarène, Gouverneur de Port Royal. Cette lettre et le détachement ont produit en partie l'effet que j'en attendois en faisant vendre le jeune abenakis enlevé par le $\mathrm{S}^{\mathrm{r}}$ Gorham ce qui a contenté les Abenakis et nous les a attachés de plus

${ }^{4}$ APC, AC, CIIA, 93 : 123-138. 
en plus. Mais il paroit par la réponse de M. Shirley que les Anglois ont dessein de soutenir leurs injustes prétentions et que par conséquent si on veut éviter une rupture ouverte en ce pays-ci on doit prendre des mesures en Europe pour arrêter leurs entreprises.

La rivière Saint-Jean n'est pas le seul poste qu'ils voudroient envahir, ils prétendent toute la côte depuis cette rivière jusqu'à Beau-bassin depuis Canceaux jusqu'à Gaspey et toute la profondeur des terres, ce qui non seulement leur donneroit contre tous les traités, plusieurs postes dans le golfe Saint Laurent, mais encore les rendroit souverains de tout le pays occupé par les Abenakis catholiques et sujets du roy, nation qui n'a jamais reconnu ni du reconnoître leur domination et qui est la plus fidèle que nous ayons en Canada. Si nous abandonnions à l'Angleterre ce terrain qui comprend plus de cent quatre-vingts lieues de côtés, c'est-à-dire presqu'autant qu'il y en a de Bayonne à Dunkerque, il faut renoncer à toute communication par terre de Canada avec l'Acadie et l'isle Royale et à tout moïen de secourir l'une et de reprendre l'autre. Il faut oter à presque tous les Acadiens toute espérance de refuge sur les terres francoises la plupart n'étant pas à portée de l'isle Saint Jean dont d'ailleurs les terres ne sont pas trop attirantes.

Par cet abandon on mettra les Acadiens au désespoir, les Anglois n'auront plus nulle raison de les ménager, ils y détruiront à leur aise la religion et n'y souffriront plus de missionnaires enfin ils empecheront la communication de l'Acadie avec Louisbourg dont la subsistance dépendra absolument d'eux.

Toutes ces vues se manifestent déjà clairement tant dans la lettre de M. Shirley que par les procédures faites à Annapolis Royale, par leurs projets de bâtir à Chibouctou, à Beaubassin et aux Mines, par les levées qu'ils font en Europe pour peupler, disent-ils, leur Nouvelle-Ecosse, enfin par toute leur conduite.

Ce n'est pas d'aujourd'hui qu'ils ont formés ces ambitieux projets; j'ai desja cité une grande carte que j'ay vue et à laquelle ils ont donné pour titre: 
l'empire anglois dans l'Amerique avec les conquêtes sur les Espagnols et sur les François.

Ils y étendent leur Nouvelle Ecosse jusqu'au fleuve Saint-Laurent dont le bord du sud leur appartiendra presque jusqu'à Quebec si on s'en rapportoit à cette carte.

Elle n'est pas aujourd'huy leur seul titre et ils font beaucoup de fond sur celle qui se trouve dans l'histoire du Canada du Révérend Père Charlevoix, dans laquelle on a copié par inadvertance une partie de cette carte angloise. Je ne dois pas dissimuler un titre beaucoup plus apparent mais que je ne connois que par les citations de leurs lettres, c'est une concession faite par Louis Treize dans laquelle on a joint à l'Acadie, toutes les cotes depuis la Nouvelle Angleterre, c'est-à-dire depuis Kinibiki jusqu'à Gaspey, mais il est manifeste que les plenipotentiaires d'Utrecht n'ont eu nulle connoissance de cette pièce, car ils n'auroient pas déclaré aussi positivement qu'ils ont fait que le golfe Saint Laurent nous appartenoit en entier.

Cette pièce obscure n'a pas pu donner au continent le nom d'Acadie connu de tout le monde pour une presqu'île et l'Acadie suivant ses anciennes $l i$ mites est la presqu'ile bornée par son isthme.

Après ces préliminaires et pendant la longue paix qui a suivi le traité d'Utrecht ils ont essayé d'établir de fait leur domination qui n'étoit qu'idéale. Ils ont escroqué de quelques sauvages par pression ou par menaces des reconnoissances contraires à la fidélité qu'ils doivent au Roy; ils en ont fait autant des habitants de la rivière Saint-Jean qui étant demisauvages eux-mêmes et ne connoissant d'autres francois que ceux de la presqu'île de l'Acadie cédée par le traité d'Utrecht ont cru devoir suivre leur sort. Je ne sais s'ils n'ont pas obtenu aussi les mêmes soumissions des habitants de Chypoudy, Pescoudiak et Mémeranconis ou du moins de quelques-uns d'eux. Ces pauvres habitants sont presque tous sortis de familles acadiennes, ils ont été presque entièrement abandonnés du Canada et de la France depuis la paix d'Utrecht et les Anglois leur ont fait entendre qu'ayant obéis ci devant au gouverneur françois de Port-Royal, ils devoient la même obeissance au gou- 
verneur anglois. Tous ces habitants qui sont en assez grand nombre n'en sont pas moins françois et places sur terre de france comme il est facile de s'en convaincre en jetant les yeux sur le traité d'Utrecht et sur toutes les cartes antérieures à ce fastueux empire anglois et même sur les livres de géographie ou l'Acadie est, ce me semble, toujours qualifiée de presqu'isle; il en est ainsi dans toutes les cartes imprimées dans leur pilote anglois quoiqu'elles soient très nouvelles et qu'elles leur donnent les unes plus, les autres moins de notre terrain mais aucune que j'ai vu n'a encore poussé la fraude jusqu'à étendre le nom d'Acadie en dehors de la presqu'isle.

Plus on étudiera cette matière, plus on verra que par le traité d'Utrecht, en cédant aux anglois l'Acadie suivant ses anciennes bornes on n'a rien cédé du continent du Canada et que les anciennes bornes de la Nouvelle France et de la Nouvelle Angleterre qui ont été posées d'un commun accord au Kinibiki en 1700 et n'ont point été ébranlées. Ainsi, bien loin que $\mathbf{M}$. de Boishébert soit sur leur terrain à Médoctet et à Menacouche ce sont eux qui pendant la dernière paix ont bâti sur le notre le fort Saint Georges près de Kinibiki et Chouaguen sur le lac Ontario et nous sommes en droit d'en demander la restitution.

Je ne dis rien sur leur récrimination contre le fort Saint Frédéric, elle n'a pas le moindre fondement étant connu de tout le monde non seulement que ce lac a été découvert par le $\mathrm{S}^{\mathrm{r}}$ Champlain avant qu'aucun anglois ou flamand en eut approché, mais encore que nous avons conquis sur les Iroquois tout ce canton et jusqu'au village des Anniers inclusivement, et que depuis plus de cinquante ans on a regardé de part et d'autre, par une convention tacite la hauteur des terres en cet endroit comme la borne entre les François et les Anglois ce que ces derniers ont du trouver alors avantageux, puisqu'en vertu de nos expéditions contre les villages Iroquois nous pouvions pousser beaucoup plus loin nos prétentions aussi quelque nuisible qu'ils aient prévu que leur serait le fort St Frédéric, ils n'ont fait que je sache aucun acte contre son établissement au lieu que $M$. 
le Marquis de Vaudreuil s'est toujours plaint du fort Saint Georges et que M. le Marquis de Beauharnois a protesté contre celui de chouaguen.

Il seroit bien à souhaiter qu'on ne s'en fut pas tenu là, surtout à l'égard de ce dernier. Il n'y a nulle apparence que l'Angleterre eut voulu soutenir ce poste, l'usurpation étoit alors trop manifeste mais enfin il ruine et ruinera de plus en plus cette colonie ci non seulement parce qu'il lui enlève une grande partie des pelleteries mais par l'esprit de révolte qu'on y inspire aux sauvages au milieu même de la paix.

Ce que je dis ici sur Chouaguen n'est pas si étranger à mon sujet qu'on le croiroit d'abord. Quoique nos raisons pour revendiquer ce poste et celui du fort Saint-Georges près de Kinibiki soient très bien fondées en justice on ne peut guère se flatter de les faire gouter en Angleterre, mais peutêtre qu'en mettant notre droit dans toute son évidence et en relachant une partie on pourroit obtenir l'autre, c'est à dire qu'en consentant que le fort Saint-Georges restât aux Anglois ils pourroient peut être nous rendre Chouaguen.

Et il est à remarquer:

Prem.: que tous les établissements maritimes tiennent toujours plus à cour aux anglois que les autres.

Deuxièmement: que Chouaguen ne dépend pas à proprement parler de la nouvelle Angleterre proprement dite (autrement Massachussett) mais de New York ou plustot d'Orange qui est celui de leurs établissements à l'Amérique à qui ils s'intéressent le moins parce qu'ils est habité principalement par des flamands que les Anglois méprisent et haïssent.

On pourrait entamer cette négociation en se plaignant en Angleterre du ton aigu dont m'écrivent Mrs Shirley et Clinton. On peut juger par mes lettres que j'ai envoyées avec les leurs si je me le suis attiré car quoique j'ai cru devoir m'opposer avec fermeté à leurs entreprises, il me semble que je l'ai fait en termes assez mesurés.

Aussi ai-je lieu d'espérer qu'à l'arrivée de ma première lettre l'affaire des prisonniers avec $M$. 
Clinton aura été décidée car elle me paroit extrêmement claire en elle même et peu interessante pour les couronnes. Il n'en est pas tout-à-fait ainsi de celle que j'ai traitée dans cette lettre-ci à laquelle je joins :

Prem.: la réponse de M. Shirley Gouverneur de Massachussett à la lettre que j'avais écrite le quinze janvier dix sept cent quarante neuf avec la traduction française.

$2^{\circ}$ l'ordonnance en anglais et en français du conseil d'Annapolis Royal contre le $\mathrm{S}^{\mathrm{r}}$ Brassard, prêtre.

$3^{\circ}$ la requête presentée à $M$. l'Evêque de Quebec par les habitants de Piziquid.

$4^{\circ}$ la lettre du $\mathrm{S}^{r}$ Brassard à $M$. l'Evêque de Québec.

$5^{\circ}$ un extrait d'un mémoire du feu $\mathrm{S}^{\mathrm{r}}$ de la Mothe Cadillac sur les limites de l'Acadie.

$6^{\circ}$ un mémoire du R. P. Germain Jésuite où il donne une idée très juste des habitations situées dans le continent de Canada et sur lesquelles les Anglois forment des pretentions.

J'observerai sur ce mémoire qu'il est fort aisé d'empecher les Anglois d'envahir ces terrains. Ils ne peuvent aller à la plupart qu'en traversant des bois ou le long des rivières fort étroites et tant que nous serons maîtres des Abenakis et que les habitants seront sûrs d'être secourus ou seulement avoués par la France, les Anglois ne s'exposeront pas à les aller attaquer chez eux. J'observerai aussi que si on prend ce party il faut avoir attention d'envoyer de Louisbourg des marchandises propres à la consommation des habitants et à la traite avec les sauvages.

Quelque longue que soit cette lettre, il faut que j'y ajoute que deux raisons principales m'ont engagé à défendre aux Abénakis aucune soumission envers le gouvernement anglois.

La première, que ce gouvernement les veut regarder comme des sujets et qu'ils sont sujets du Roy.

La deuxième c'est que $M^{r}$ Clinton n'a jamais voulu souffrir que les Anniers vinssent ici faire des soumissions pour ravoir leurs prisonniers. Je n'ai cependant jamais prétendu que les anniers recon- 
nussent le roy pour leur souverain, quoique nous le puissions prétendre avec plus de droits que les Anglois n'en ont sur les Abénakis.

$\mathrm{Au}$ reste je ne suis pas le premier commandant ou gouverneur général de Canada qui me sois opposé depuis le traité d'Utrecht aux prétentions des anglois sur la rivière Saint Jean et je le puis prouver par bien des titres mais je me contente de la copie ci-jointe d'une lettre de M. Le Marquis de Vaudreuil à M. Dummer gouverneur de Boston. Je ne diffère de lui qu'en une chose à quoy il me paroit essentiel de faire attention c'est que par des ménagements qui lui étoient prescrits et dont les raisons ne subsistent plus aujourd'hui à ce que je crois, il n'attaquoit les usurpations angloises que comme faites sur les terres abenakises, au lieu que j'ai cru devoir les attaquer comme faites sur le terrain françois. La première de ces tournures si on continuoit à s'en servir tendroit à rendre équivoque notre droit qui me paroit incontestable, ou du moins le feroit dépendre de la nation abenakise.

Je crois donc qu'on doit persister dans le même sans abandonner l'autre et que si les circonstances ne permettent pas d'en tirer avantage à present ou interrompre du moins par là la prescription en attendant quelque occurence plus favorable. Je finis en avertissant que je ne suis pas suffisamment instruit, n'ayant actuellement presque point de livres et très peu de titres. J'ai l'honneur d'être avec un très profond respect, Monseigneur, votre très humble et très obéissant serviteur.

\section{La Galissonière.}

Le document que nous venons de lire présente un certain nombre de données qui furent l'objet de discussions francobritanniques. Un échantillonnage des pièces versées au dossier des négociations permet de souligner quelques difficultés qui ont surgi au cours de ces rencontres diplomatiques.

La Galissonière et Silhouette sont investis des pleins pouvoirs de négociation relative à "toutes les prétentions réciproques tant au sujet des isles de Tobago, Sainte-Lucie, Saint-Vincent et la Dominique que par rapport aux limites des colonies res- 
pectives dans l'Amérique septentrionale". Ils ont aussi mission d'étudier "les contestations relatives aux prises induement faites à la mer de part et d'autre à l'occasion de la guerre". ${ }^{5}$

A la première conférence, les Britanniques représentés par Mildmay et Shirley voulaient discuter la question des frontières acadiennes, tandis que les Français désiraient y ajouter le problème de la possession de Sainte-Lucie. Le refus des Britanniques a incité les représentants français à proposer de consacrer, chaque semaine, une journée à cette double étude. Selon les Anglais, l'évacuation de Sainte-Lucie était préalable à toute discussion relative au droit de possession.

La troisième conférence indique l'axe de développement des négociations. Les réclamations britanniques concernant l'Isle Royale et celle de Saint-Jean ainsi qu'une partie du golfe et la rive gauche du fleuve Saint-Laurent sont équivalentes à une demande de cession du Canada, aux yeux des délégués français.

Le 28 septembre 1750, à la quatrième conférence, les Anglais n'avaient pas l'intention de hâter les procédures. Ils firent savoir qu'ils n'avaient pas reçu l'autorisation de discuter la double question de Sainte-Lucie et des limites de l'Acadie. Ils ont suggéré d'inviter les Hollandais aux discussions de frontières. Les commissaires français répondent qu'ils n'ont pas le pouvoir de traiter avec cette nation.

Lors de la sixième conférence, La Galissonière et Silhouette font part au ministre Puyzieulx d'un délai dans la communication des instructions que les délégués anglais attendaient de leur métropole au sujet de Sainte-Lucie et des frontières acadiennes. Ils se méfient de ce retard qui peut avoir, à leur avis, des suites fâcheuses. "Nous avons été arrêtés par la représentation que ces Messrs. nous ont faite, qu'ils n'avoient pas de pouvoir à cet égard."

Accueillies avec réserve et appréhension, les propositions des commissaires de la France et de la Grande-Bretagne suscitèrent de longues discussions qui aboutirent à un échec.

5 APC, Affaires Etrangères. Correspondance politique, vol. 430-431. 


\section{LA MARINE, AGENT DE CIVILISATION TECHNIQUE}

La transformation industrielle débute en Grande-Bretagne, au seuil du XVIII siècle. Elle se fonde sur l'application de la méthode expérimentale, dénominateur commun des découvertes et des inventions. Inscrite dans le cadre des exigences humaines de civilisation qui se définit par les manifestations d'ordre intellectuel, artistique et religieux et par un ensemble d'institutions politiques et de conditions matérielles, la révolution industrielle influe sur la société qui, en raison de l'augmentation des besoins collectifs, exerce une pression accrue sur l'entreprise de diversification des moyens d'action technique.

Les progrès scientifiques accompagnent la diffusion de "l'esprit du siècle". Selon Charles Morazé, "c'est à partir du XVIII ${ }^{\mathrm{e}}$ siècle que la science est liée au bonheur des peuples, que le destin des civilisations se décide dans les laboratoires." " $\mathrm{De}$ nombreux souverains d'Europe encouragent les sciences et leurs applications par la fondation de sociétés savantes qui favorisent des échanges dans une ambiance de cosmopolitisme et un courant d'idées de progrès. Les disciplines d'observation directe de la nature et les méthodes expérimentales suscitent la curiosité des maîtres de la pensée et des membres de l'aristocratie et de la classe politique. Voltaire, notamment, vulgarise en France les théories scientifiques de Newton.

Dès la fin du XVII ${ }^{e}$ siècle, l'Angleterre s'imposa comme le centre le plus actif de fabrication d'instruments scientifiques. Elle a pris un tel essor que sa prépondérance se maintint jusqu'à la disparition de l'artisanat et à l'avènement de la production en série. En Angleterre, où s'accumulent les capitaux disponibles, se produisit une transformation industrielle, d'abord lente, qui s'accélère au cours du XVIII ${ }^{\mathrm{e}}$ siècle.

L'industrie française ne pouvait pas se passer du concours direct de l'Etat qui drainait une grande partie des capitaux. Les progrès techniques furent plus lents qu'en Grande-Bretagne. "La Marine fut sacrifiée à la nécessité de défendre nos fron-

${ }^{6}$ René Taton, éd., La Science Moderne, Histoire Générale des Sciences (Paris, 1958), 431. Voir John U. Nef, Les Fondements culturels de la civilisation industrielle (Paris, 1964). 
tières", affirme Condorcet. Faute de fonds, le cardinal de Fleury et le ministre Maurepas ont laissé "la Marine dans l'état de foiblesse" où ils l'avaient trouvée. ${ }^{7}$

La construction navale nécessite un ensemble d'industries complémentaires: le charbon, le bois, le fer, le goudron, les voiles et cordages, les instruments d'astronomie nautique. La Marine joue au XVIII ${ }^{\mathrm{e}}$ siècle le rôle d'agent de colonisation qui sera, au siècle suivant, celui des chemins de fer. La primauté que les Britanniques avaient acquise dans le domaine industriel a ses effets en Nouvelle-France, avant le changement d'empire. Louis-Antoine de Bougainville expose, en 1758, un aspect de dépendance économique et technique: "On a besoin en Canada des petits batimens pour les postes de la pêche, pour le commerce de Québec à Montréal, pour le cabotage de la rivière, pour la traite à Gaspé et à Louisbourg, et cette partie de la construction est si fort négligée icy que les Anglais de ce continent fournissent une partie des batimens pour la navigation dans l'intérieur de notre colonie, ce n'est pas que leurs bois soient meilleurs, ou leurs batimens mieux construits que les nôtres, mais ils les donnent à meilleur marché, aussi voyons nous dans toutes nos places maritimes des navires marchands construits dans la Nouvelle-Angleterre." 8

La montée du potentiel technique provoque une réaction à chaînes qui atteint tous les facteurs de civilisation, sous forme d'interaction. Elle suscite, notamment au sein des classes sociales, un dynamisme propre à la formation de nouvelles attitudes de pensée liée au goût de la précision et à l'habitude de gestion d'entreprises de transformation.

"La France, écrit Cordorcet, a produit plus d'ouvrages importans sur la Marine, que l'Angleterre même: et comment sans les avantages de cette union entre les sciences et l'art nautique, union également nécessaire et plus nécessaire qu'on ne croit aux progrès de tous les arts, un peuple dont la Marine ne peut être ni le premier objet, ni la principale occupation,

${ }^{7}$ Histoire de l'Académie royale des Sciences (Paris, 1781), 82 et 83.

8 Bougainville, Considérations sur l'état présent du Canada, Mss. 19, Archives du Séminaire de Québec. 
aurait-il pu s'élever au niveau d'une nation qui la regarde comme sa seule puissance et la source de ses richesses !" 9 Même si la France rivalise avec la Grande-Bretagne sur le plan de la poursuite de l'examen expérimental des phénomènes physiques, elle n'a pas transposé, au même rythme qu'en Angleterre, les résultats scientifiques dans le domaine de production, et en particulier, dans celui de la construction navale.

Sur le terrain de rivalité pour l'ambition impérialiste, la régression d'une métropole par rapport à sa concurrente dans le secteur des industries de transformation et d'équipement technique a pour effet de mettre en péril une colonie située au voisinage de la puissance dominante, car le destin colonial se joue dans la métropole. Si l'accent porte sur l'activité maritime qui sert de support à bien des industries connexes ou utilisatrices, il n'en est pas moins vrai que l'implantation industrielle est liée au facteur démographique, comme le rappelle La Galissonière dans une lettre adressée à l'Inspecteur général de la Marine, Duhamel du Monceau, le 28 octobre 1748, et conservée à l'American Philosophical Society.

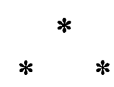

Les détails entrevus dans cette étude sont révélateurs de la complexité de la situation coloniale au milieu du XVIII ${ }^{\mathrm{e}}$ siècle. Ces problèmes exigent de ceux qui en poursuivent l'examen un effort de recherche sur l'ensemble des faits de civilisation et de milieu géographique, pour réunir les éléments d'appréciation historique. Ce domaine d'interdépendance des disciplines révèle les limites de la recherche individuelle; il suscite entre les spécialistes une prise de conscience du fructueux apport du travail en équipe. Soucieux de se régler sur sa propre discipline, le chercheur en histoire profite d'une participation, au sein d'une œuvre de coordination, à la mise en commun des résultats d'enquête fournis par les sciences auxiliaires de l'histoire.

Université de Montréal.

ROLAND LAMONTAGNE

${ }^{9}$ Histoire de l'Académie royale des Sciences (Paris, 1781), 87. 